\title{
Probalandia, un escenario de juegos de azar para la enseñanza de las técnicas de conteo y el concepto básico de probabilidad ${ }^{1}$
}

\author{
Probalandia a gaming scenario for teaching counting \\ techniques and basic concept of probability
}

Probalandia um cenário de jogos para o ensino de técnicas de contagem e conceito básico de probabilidade

Recibido: mayo 2013

Aceptado: agosto 2013
Diana Paola Piedra Moreno ${ }^{2}$

Cristian Gonzalo Camacho Ruiz ${ }^{3}$

\section{Resumen}

Se asume la teoría de situaciones didácticas como forma de orientar el proceso de aprendizaje de los escolares, la cual plantea que no es posible transmitir el saber al estudiante de forma directa sino que es necesario recrear una micro-cultura del hacer matemático para que los alumnos requieran solucionar y adaptarse a problemáticas de un medio. Por ello se presenta una experiencia que consistió en recrear un escenario llamado Probalandia (centro de entretenimiento famoso por ejecutar cuatro juegos de azar que eran justos, pero que perdió credibilidad cuando se murmuró que ya no lo eran) en el cual estudiantes de $11^{\circ}$ encontraron juegos justos e injustos y con ello aprendieron técnicas de conteo y el concepto básico de probabilidad.

Palabras clave: Azar; matemáticas escolares; estadística; probabilidad; enseñanza de las técnicas de conteo; concepto básico de probabilidad.

\begin{abstract}
Theory is assumed didactical situations as a way to guide the learning process of the students, which states that no knowledge can be transmitted directly to the student it is necessary to recreate a micro-culture of doing math for students and adapted to solve existing problems of a medium. Therefore presents an experience that was to recreate a scenario called Probalandia (famous entertainment center run four gambling that they were righteous, but lost credibility when he muttered that they were not already) in which students from $11^{\circ}$ found fair and unfair games and thus learned counting techniques and basic concept of probability.
\end{abstract}

Keywords: school mathematics, statistics, probability, teaching counting techniques, basic concept of probability.

1 Artículo de Investigación

2 Universidad Distrital Francisco José de Caldas. Bogotá, Colombia. Contacto: diath_93@hotmail.com

3 Universidad Distrital Francisco José de Caldas. Bogotá, Colombia. Contacto: cristiangcamachor@hotmail.com 


\section{Resumo}

Teoria é assumido situações didáticas, como forma de orientar o processo de aprendizagem dos alunos, que afirma que nenhum conhecimento pode ser transmitido diretamente para o aluno é necessário recriar um micro-cultura de fazer a matemática para os alunos e adaptado para resolver os problemas existentes, de um meio. Portanto, apresenta uma experiência que era recriar um cenário chamado Probalandia (famoso centro de entretenimento executar quatro jogos de azar que eram justos, mas perdeu credibilidade quando ele murmurou que não eram já) em que os alunos de $11^{\circ}$ encontrados justo e injusto jogos e, assim, aprendeu técnicas de contagem e conceito básico de probabilidade.

Palavras-chave: Azar; matemática escolar, estatística, probabilidade, ensinando técnicas de contagem, o conceito básico de probabilidade.

\section{Contextualización}

De acuerdo con el Ministerio de Educación Nacional (2006) es importante la enseñanza del pensamiento estocástico, porque brinda argumentación en situaciones de incertidumbre, azar y riesgo y porque es una forma de relacionar el azar y la realidad de la vida del hombre (Batanero y Godino, 2002). Siguiendo con lo anterior, se asumen los juegos de azar como una realidad de la vida del hombre y que son cercanos a los estudiantes por tratarse de una actividad de ocio o negocio que puede ser estudiada a partir de las técnicas de conteo para cuantificar acciones favorables para el jugador, dichas cuantificaciones permiten llegar de forma intuitiva al aprendizaje del concepto básico de probabilidad (Fischbein, 1975 citado en Batanero, 2013).

Se observa la importancia del proceso de enseñanza-aprendizaje del concepto básico de probabilidad empleado técnicas de conteo, pero ¿Qué se puede decir de la realidad en las escuelas de Colombia? Para responder esta cuestión Garzon y Forero (2009) indican que dentro de los estándares curriculares la probabilidad está estipulada como tópico de enseñanza pero las prácticas de aula no lo hacen evidente debido a desconocimiento de formas de enseñanza o en ocasiones resulta ser trabajada en un corto tiempo y de forma mecánica (aplicación de formulas que carecen de sentido). Bajo este panorama se hizo necesario escribir una experiencia de aula para el trabajo con las técnicas de conteo y del concepto básico de probabilidad, como medio de difusión de una forma de enseñanza bajo una metodología para el aprendizaje significativo.

\section{Referentes teórico-prácticos básicos.}

La experimentación debe ser medio para que el estudiante considere necesario medir la incertidumbre para la toma de decisiones, este tipo de medición debe efectuarse sin recurrir a la frecuencia de las consecuencias observadas, pasando al uso de las técnicas de conteo, acordes a cada situación (diagrama de árbol, principio multiplicativo, permutación y combinación) y que le permiten tener en cuenta los eventos favorables, en contra y el total de eventos posibles.

De esta manera se hace necesario asumir algunas consideraciones para cuando se trabaja con técnicas de conteo, por ejemplo el diagrama de árbol constituye un instrumento útil a la hora de comprender las operaciones posteriores con las cuales se podría abreviar algunos procesos, pero su uso irreflexivo puede conllevar a la creencia de los estudiantes de considerarle siempre pertinente para encontrar el cardinal de combinaciones de elementos que se pidan. Por ello es importante enseñar y generar un choque en los estudiantes con situaciones en las cuales se requiera medir un cardinal relativamente 
grande, e interpreten que no siempre resulta efectiva dicha técnica (Godino, Batanero y Cañizares, 1996).

El diagrama de árbol también constituye un elemento para efectuar el cambio de una operación concreta (establecer cuáles son la combinaciones) a una formal (establecer cuantas, por medio de la aplicación del principio multiplicativo) según Fischbein (1975, citado en Batanero, 2013) De acuerdo con lo anterior, se requiere analizar ese paso de lo concreto a lo abstracto, esto será el insumo para el diseño e implementación de la propuesta, para ello se usan las etapas de Dienes (1982, citado en Godino y otros, 1996) quien caracteriza precisamente este hecho. Él propone seis etapas las cuales se relacionan con el trabajo en Probalandia:

Interacción inicial: en esta etapa se realiza experimentos con juegos de azar, por ello se interactúa con un medio que también puede arrojar respuestas. Pero en esta etapa, el estudiante permanece únicamente en la interacción sin una reflexión acerca de lo que experimenta.

Descubrimiento de regularidades: el estudiante reflexiona acerca del experimento y usa las frecuencias de ocurrencia de diferentes eventos como forma de plantear la existencia de la probabilidad en relación a las partidas jugadas y a las ganadas. Descubrir regularidades también implica el uso de representaciones, inicialmente la representación en diagrama de árbol. 3 - Búsqueda de isomorfismos: se da con el uso de dispositivos isomorfos "par o impar", "ganar o perder"...etc.

Representación: en esta etapa se da el uso de tablas, diagramas de sectores o de análisis de eventos por secciones, combinaciones y permutaciones.

Propiedades de representación: se encuentra propiedades de la probabilidad, como "la suma de probabilidades de un mismo sistema da 1 y una probabilidad puede ser mayor, menor o igual que otra", la probabilidad se expresa con números reales entre 0 y 1 , por ello lo justo es tener la misma probabilidad de ganar que de perder (igual a 0,5 ) o la misma probabilidad para todos los jugadores.
Formalización del sistema: en esta etapa se usa la representación formal del cálculo de probabilidades, como razón o cociente entre eventos favorables y el total de los posibles.

En cuanto a esta última etapa, se considera pertinente mencionar que según Godino, Batanero y Cañizares (1996) la probabilidad como razón puede ser interpretada desde la percepción (razón entre lo a favor y en contra), que debe ser superada para llegar a la concepción básica (razón entre la cantidad de eventos favorables, sobre la cantidad total de eventos posibles), ya que muchos estudiantes consideran que la probabilidad hace referencia a la primera razón mencionada, esta dificultad es frecuente. (Fischbein, 1975 citado en Batanero, 2013)

En cuanto a la organización de las sesiones de clases, teniendo en cuenta la teoría de situaciones didácticas se trabajó con una situación fundamental (Probalandia). En cada actividad de la secuencia de actividades, existió al menos una tipología de situaciones, esto quiere decir según Panizza (sf) que se llevaron al aula las diferentes clasificaciones de la situación en acción, formulación, validación e institucionalización. Panizza explica que la situación de acción es aquella en la cual el estudiante tiene un momento personal para interactuar con el medio; la situación de formulación en la cual se van encontrando algunas regularidades arrojadas por el medio, gracias a la comunicación entre estudiantes; la situación de validación en la cual se va enunciado premisas que se van cuestionando como verdaderas o falsas, basadas en argumentos. Finalmente está la situación de institucionalización en la cual con ayuda del maestro, se establecen acuerdos comunes y culturales entre los estudiantes, en relación al saber puesto en juego (concepto básico de probabilidad) el cual se vuelve explícito.

\section{Descripción general de la experiencia de aula}

Considerando la Teoría de situaciones didácticas, a modo de descripción se enseña la situación 
fundamental y lo que se hizo en cada fase, todo el proceso se efectuó en nueve sesiones de clases de dos horas.

Situación. Probalandia es un lugar para disfrutar de diferentes juegos, este centro de distracción ha sido famoso y de los más visitados por ser el único en manejar juegos justos. Hace un tiempo perdió su fama, lamentablemente se empezó a escuchar el rumor de que sus juegos ya no eran justos. Los dueños de Probalandia un tanto preocupados por esta situación, le han solicitado al grado once del colegio José Félix Restrepo, que encuentre los juegos injustos, para no dejarlos volver a funcionar y así salvar su empresa de la quiebra. De esta manera los estudiantes de grado once requieren descubrir: ¿CUÁLES JUEGOS SON INJUSTOS Y POR QUÉ? Para iniciar con la labor, se les entregó el listado de juegos disponibles en Probalandia.

Tabla 1

El sexteto de dados: en este juego hay dos secciones, el jugador lanza seis dados, en la sección a gana si obtiene las seis caras con números diferentes y en la sección $\mathrm{b}$ gana si se obtiene dos números iguales y el resto diferentes.

¿Es este juego injusto en la sección a, por qué? ----------- ¿Es este juego injusto en la sección b, por qué?

El juego por colegio: en este juego participa todo un curso de colegio, Probalandia pide que del curso 1103 que tiene 33 estudiantes se escoja de forma justa una sola pareja que participará en representación del curso. Luego de escoger la pareja, se informa que el curso gana sí escogió efectivamente una pareja de forma justa. ¿Es este juego injusto, por qué?

Balotas games: en este juego hay una bolsa que oculta 6 balotas rojas y 6 amarillas, gano si saco un par de balotas del mismo color. ¿Es este juego injusto, por qué?

El colodado: en este juego hay dos secciones, existe un dado numérico (con números del 1 al 6 ) y uno de color (mitad azul y mitad rojo), lanzo primero el numérico, luego el de color, si saco azul multiplico el número del dado por 2 y si saco rojo lo multiplico por 3. En la sección a, gano si el producto es un número impar. En la sección b, gano si el producto es un múltiplo de 7.

¿Es este juego injusto en la sección a, por qué? ------- ¿Es este juego injusto en la sección b, por qué?

Fuente: ealboración propia

Trabajo en cada fase. en el Anexo 1 se enseña una tabla que resume las temáticas y el producto de trabajado en relación a cada fase.

\section{Logros y dificultades evidenciadas}

Con la situación de Probalandia, los estudiantes pasaron de emplear técnicas de conteo no convencionales (estimación: mucho y poco) a reconocer su aplicación en determinados contextos, como forma de cuantificar eventos y llegar al concepto básico de probabilidad, pasando poco a poco de su uso informal (imposible, seguro, cuestión de suerte) a una idea intuitiva (razón entre los eventos a favor y en contra) y llegando posteriormente al concepto básico de probabilidad (razón entre lo favorable y lo posible). Una dificultad evidenciada fue el cansancio estudiantil por trabajar la misma situación durante varias sesiones de clase, pese a tener trabajos diferenciados.

\section{Reflexión final}

A modo de reflexión se estipula que es importante diseñar situaciones fundamentales que no desgasten al estudiante por tomarle en su solución varias sesiones de clase. Por otro lado la experiencia de aula permitió generarle a los docentes algunos cuestionamientos respecto a la evaluación bajo la metodología que se asumió ya que se evaluaron únicamente los registros escritos pero se notó que la expresión oral también constituye una fuente para hacer un pronunciamiento del proceso de aprendizaje de los estudiantes.

\section{Referencias}

Batanero, C., \& Godino, J. (2002). Estocástica y su didáctica para maestros. Granada: Repro Digital. 
Batanero. C. (2013). La Comprensión de la Probabilidad en los niños: ¿Qué Podemos Aprender de la Investigación?

Garzon, A., \& Forero, M. (2009). Diseño de una secuencia de actividades para la enseñanza de la probabilidad simple en estudiantes de sexto grado. Aplicación y validación. Conferencia presentada en $10^{\circ}$ Encuentro Colombiano de Matemática Educativa , 10, 02-03.
Godino, Batanero, \& Cañizares. (1996). Azar y probabilidad. Madrid: Síntesis, S.A.

MEN. (2006). Estandares básicos de competencias en matemáticas. Colombia: Magisterio.

Panizza, M. (sf). Conceptos básicos de la teoría de situaciones didácticas.

\section{ANEXO 1}

\begin{tabular}{|c|c|c|}
\hline FASE & TEMÁTICA & PRODUCTO DEL TRABAJO EN CLASE \\
\hline Diagnóstico & $\begin{array}{l}\text { Principio aditivo, principio } \\
\text { multiplicativo, formas de contar y } \\
\text { concepto básico de probabilidad. }\end{array}$ & $\begin{array}{l}\text { Permitió que los estudiantes exteriorizaran sus conocimientos previos, } \\
\text { para identificar el uso del principio aditivo y multiplicativo y las formas } \\
\text { para enumerar eventos. }\end{array}$ \\
\hline A-didáctica & Formas de contar los eventos. & $\begin{array}{l}\text { Logró que los estudiantes reconocieran que para determinar si el juego es } \\
\text { justo, se requiere contar en un inicio las posibilidades de ganar, en } \\
\text { comparación a las de perder. Por ello necesitaron formas de contar los } \\
\text { eventos. } \\
\text { Ejemplo: juego Balotas games. } \\
\text { Izquierda: "el juego es equilibrado por q' hay } 6 \text { pelotas amarillas y } 6 \text { rojas" } \\
\text { Derecha: el juego es justo porque hay la misma posibilidad de ganar o perder" } \\
\text { Evidencia: En esta respuesta se reconoce la justicia como la misma posibilidad de ganar o de } \\
\text { perder (igual) los estudiantes se basan en la probabilidad de sacar alguna bola amarilla o roja ya } \\
\text { que hay } 6 \text { bolas rojas y } 6 \text { bolas amarillas. }\end{array}$ \\
\hline Acción & $\begin{array}{l}\text { Tabulación y análisis de eventos } \\
\text { de azar tras la participación en } \\
\text { partidas finitas de cada juego. } \\
\text { Técnicas de conteo (diagrama de } \\
\text { árbol, combinación, permutación) } \\
\text { Usos informales de la } \\
\text { probabilidad (imposible, } \\
\text { probable, seguro... ganar o } \\
\text { perder). }\end{array}$ & $\begin{array}{l}\text { Consiguió que los estudiantes durante la interacción con el medio } \\
\text { (experimentación en cada juego) que algunos juegos es seguro, imposible } \\
\text { o probable ganar, con ello se refirieron a la } \\
\text { justicia o injusticia. } \\
\text { Ejemplo: juego El Colodado: } \\
\text { Palabras del estudiante: "El juego en la sección B es injusto, } \\
\text { pues es imposible ganar, ya que los dados no dan dicha } \\
\text { posibilidad de ganar, porque es de } 6 \text { números del } 1-6 \text { y eso } \\
\text { se multiplica por } 2 \text { o } 3 \text { y ninguno da múltiplo de } 7 \text { " } \\
\text { Evidencia: el estudiante con este desarrollo, estudia todas } \\
\text { las posibilidades del juego Colodado, esto conlleva un } \\
\text { diagrama de árbol o el total de opciones que enmarca el } \\
\text { principio multiplicativo, específicamente en análisis de eventos por secciones, donde identifica } \\
\text { los resultados del dado numérico y como se relaciona con el dado de color. }\end{array}$ \\
\hline $\begin{array}{l}\text { Formulación y } \\
\text { comunicación }\end{array}$ & $\begin{array}{l}\text { Técnicas de conteo. } \\
\text { Concepto básico de probabilidad. }\end{array}$ & $\begin{array}{l}\text { Hizo que la situación de Probalandia, generara la formulación de que para } \\
\text { que un juego de azar fuera justo, la probabilidad de } \\
\text { ganar debía ser la misma de perder, usando el } \\
\text { concepto básico de probabilidad. } \\
\text { Ejemplo: trabajo en grupo y el aporte de los integrantes del mismo en } \\
\text { búsqueda de comparar diferentes probabilidades para saber si son justas } \\
\text { (cada jugador con la misma probabilidad) o injustas, (alguna mayor o } \\
\text { menor). }\end{array}$ \\
\hline Validación & $\begin{array}{l}\text { Eventos posibles. } \\
\text { Eventos favorables. } \\
\text { Concepto básico de probabilidad. }\end{array}$ & $\begin{array}{l}\text { Generó que los estudiantes aportaran significativamente, argumentos } \\
\text { matemáticos relacionados con el principio básico de probabilidad, para } \\
\text { formular y comprobar cuando un juego de azar era justo o injusto } \\
\text { (probabilidad de perder contra la probabilidad de ganar). }\end{array}$ \\
\hline Institucionali-zación & $\begin{array}{l}\text { Diagrama de árbol, permutación } \\
\text { (con y sin repetición) y } \\
\text { combinación. } \\
\text { Eventos posibles. } \\
\text { Eventos favorables. } \\
\text { Concepto básico de probabilidad. }\end{array}$ & $\begin{array}{l}\text { Permitió que los estudiantes aportaran a la comprensión, consolidación y } \\
\text { explicación de los temas aprendidos gracias a Probalandia. } \\
\text { Evidencia: Los estudiantes de este grupo usan diferentes técnicas de conteo, factibles en cada } \\
\text { juego. El ejemplo para el juego Colodado se analiza con el diagrama de árbol que pasa con cada } \\
\text { número si se saca azul o rojo. Y luego mediante ese conteo se realiza el cociente entre los eventos } \\
\text { favorables y los eventos posibles del experimento, utilizando esto último como la definición del } \\
\text { concepto básico de probabilidad. }\end{array}$ \\
\hline Evaluación & $\begin{array}{l}\text { Diagrama de árbol, permutación y } \\
\text { combinación. Eventos posibles. E } \\
\text { ventos favorables. Concepto } \\
\text { básico de probabilidad. }\end{array}$ & $\begin{array}{l}\text { Posibilitó elaborar un juicio comparativo y sumativo, en relación a lo } \\
\text { obtenido en el diagnóstico para determinar el progreso de cada estudiante } \\
\text { gracias a la situación fundamental de Probalandia. }\end{array}$ \\
\hline
\end{tabular}

Fuente: ealboración propia 